\title{
Resveratrol induces apoptosis in human melanoma cell through negatively regulating Erk/PKM2/Bcl-2 axis
}

This article was published in the following Dove Press journal:

OncoTargets and Therapy

\author{
Hailong Zhao' \\ Limin Han' \\ Yi Jian ${ }^{2}$ \\ Yuntao $\mathrm{Ma}^{3}$ \\ Wanyue Yan $^{3}$ \\ Xiaowen Chen' \\ Haiyan $\mathrm{Xu}^{\prime}$ \\ Lijuan Li'
}

'Department of Pathophysiology, Zunyi Medical University, Zunyi,

Guizhou Province 563000, People's Republic of China; ${ }^{2}$ The First Clinical Institute, The School of Medicine and Science, Zunyi Medical University, Zunyi, Guizhou Province 563000, People's Republic of China; ${ }^{3}$ The First Clinical Institute, The Department of Clinical Medicine, Zunyi Medical University, Zunyi, Guizhou Province 563000, People's Republic of China

Correspondence: Hailong Zhao Department of Pathophysiology, Zunyi Medical University, No 20I, Dalian Road, Huichuan District, Zunyi, Guizhou Province 563000, People's Republic of China

$\mathrm{Tel}+8685128643626$

Fax +86 85I 28642444

Email hailongzhao@zmc.edu.cn
Background: Resveratrol is known as a natural phytoalexin found in grapes and wine, which has significant antitumor activity under in vitro and in vivo conditions. In recent years, great progress has been made in understanding the underlying mechanisms of resveratrol in inducing cellular apoptosis of melanoma cells. Our previous study has shown that the apoptosis regulation of resveratrol in melanoma cells was independent of activation of classical apoptosis-related protein $\mathrm{p} 53$.

Materials and methods: MTT assay and 5-bromo-2'-deoxyuridine staining assay were used to analyze cell viability and proliferation. Immunofluorescence analysis of $\gamma$-H2AX was employed to clarify DNA damages. Annexin V-propidine iodide/fluorescein isothiocyanate assay was performed to evaluate the cell apoptosis. The mechanisms underlying the activation of M2-type pyruvate kinase (PKM2) by Erk1/2 to stabilize and maintain Bcl-2 signaling was investigated by subcellular fractionation analyses, immunofluorescence analysis, co-immunoprecipitation assay, ubiquitination assay, and glutathione S-transferase pull-down assay.

Results: In the present study, we found that resveratrol dramatically inhibited melanoma cell proliferation and induced cell apoptosis through upregulation of p53 in a concentration-dependent manner. Conversely, p53 downregulation by short hairpin RNA couldn't rescue resveratrolinduced cell proliferation inhibition or apoptosis enlargement. Additionally, we found that resveratrol downregulated antiapoptotic protein Bcl-2 and activated Bax in the protein levels by promoting Bcl-2 degradation and cytochrome c release. Moreover, we discovered that PKM2, had a key role in cell apoptosis triggered by resveratrol through interacting with Bcl-2. Based on these results, we overexpressed PKM2 in melanoma cells and found that this prevented resveratrol-induced apoptosis by stabilizing the protein level of Bcl-2.

Conclusion: Taken together, our results provided a novel mechanism accounting for the apoptosis induction of resveratrol in melanoma cells and suggested that downregulating Erk/PKM2/ Bcl-2 axis appears to be a new approach for the prevention or treatment of melanoma.

Keywords: antitumor, ubiquitination, cytochrome c, ER stress

\section{Introduction}

Malignant melanoma is one of the most often diagnosed cancers originating from melanocytes. ${ }^{1}$ Over the past decades, various medical therapies for melanoma patients including surgery, radiation, and chemotherapy have been well applied, but the incidence of malignant melanoma is still increasing and the prognosis remains extremely poor. ${ }^{2}$ The few reasons for this are as follows: 1) surgical resection is useless for melanoma patients because of undetectable lesions process and numerous organs dissemination, such as brain, lung, or liver;,3, 2) in radiation there is an urgent need to exploit the 
efficacy of the anti-melanoma therapies owing to the damage to normal physiological mechanism and immune system; ;,4,5 and 3 ) the incapacity of most chemotherapeutic drugs for high efficiency as a result of the intrinsic resistance to apoptosis in melanoma. ${ }^{6,7}$ Among these three therapies, chemotherapeutic therapy is relatively more controlled and easier to update. ${ }^{8,9}$ Therefore, it is time to investigate novel drugs for melanoma treatment with minimal toxicity and resistance.

Although melanoma is a historically treatment-resistant malignancy, recent reports showed that antiapoptotic factors from intrinsic apoptosis pathways become potential targets for overcoming the apoptotic resistance of melanoma. ${ }^{7,9,10}$ As per our previous research, NAMPT/E2F2/SIRT1 signaling pathway among the nicotinamide adenine dinucleotide (oxidized) $\left(\mathrm{NAD}^{+}\right)$-dependent regulation network in human melanoma cells regulated cell proliferation and apoptosis resistance. However, inhibition of the NAMPT/E2F2/SIRT1 pathway was involved in but independent of p53 activation. ${ }^{11}$ At the same time, resveratrol used as a potent activator of SIRT1 through AMP-activated protein kinase induction could not affect the stimulating apoptosis-regulated protein p53 expression. ${ }^{11,12}$ Eventually, even resveratrol-induced upregulated SIRT1 indirectly methylated $\mathrm{H} 3 \mathrm{~K} 9$ in the nucleus and deacetylated p53 in the cytoplasm to inhibit p53 and abrogate apoptosis. ${ }^{12}$

Nevertheless, many studies have demonstrated that resveratrol suppresses cancer cell growth and proliferation through inducing cellular apoptosis. ${ }^{10,13,14}$ In the intrinsic pathway of mammalian cells, DNA damage, hypoxia, and growth factor inhibitor activate the Bcl-2 family members (Bax and Bak) and result in the mitochondrial release of cytochrome $\mathrm{c}$ to induce apoptosis. ${ }^{10,15} \mathrm{Bcl}-2$ family is essential for apoptosis regulation and the first apoptotic regulator is identified as an oncogene. ${ }^{6}$ Numerous chemotherapeutic inhibitors of Bcl-2 family proteins as therapeutic targets have been generated. Consequently, Bcl-2-mediated apoptotic pathway may be another possible association with resveratrol-induced apoptosis in melanoma cells except for p53related apoptotic pathway.

To track what mechanism is involved in resveratrol-induced apoptosis, we chose some possible target genes related to resveratrol treatment for further research. First, Wu et al identified that resveratrol induced cancer cell apoptosis targeting M2-type pyruvate kinase (PKM2)-mediated ER stress and mitochondrial fission. ${ }^{14}$ Second, Yang et al and Wu et al evaluated that ERK1/2-dependent nuclear translocation of PKM2 promoted glucose metabolism in various cancer cells. ${ }^{16,17}$ Finally, Liang et al reported that mitochondrial PKM2 interacted with and phosphorylated Bcl-2, which then prevented the degradation of Bcl-2 and enhanced apoptosis resistance of tumor cells. ${ }^{18}$

In light of these previous findings, we hypothesized that resveratrol suppressed melanoma proliferation through inducing human melanoma apoptosis by downregulating $\mathrm{Bcl}-2$ rather than upregulating $\mathrm{p} 53$. In this paper, our research first put forward that resveratrol has anti-melanoma activity through disrupting PKM2-Bcl-2 interaction and promoting Bcl-2 degradation for impairing melanoma cells growth. Further, these results demonstrated that elevated PKM2 resulted in offsetting resveratrol inducing melanoma cell proliferation arrest and apoptosis by recovering the protein level of Bcl-2, highlighting the potential of resveratrol and PKM2 in the treatment of metastatic melanoma.

\section{Materials and methods Cell culture and treatment}

Human melanoma cell lines (MV3 and A375) and human embryonic renal cell line 293FT were obtained from the American Type Culture Collection (ATCC), Manassas, VA, USA. Cell line MV3 was maintained in Roswell Park Memorial Institute-1640 (Thermo Fisher Scientific, Waltham, MA, USA) medium. A375 was cultured in DMEM (Thermo Fisher Scientific). Media was supplemented with 10\% FBS, $2 \mathrm{mg} / \mathrm{mL}$ glutamine, $100 \mathrm{U} / \mathrm{mL}$ penicillin, and $100 \mu \mathrm{g} / \mathrm{mL}$ streptomycin (Sigma-Aldrich Co., St Louis, MO, USA). Cells were incubated at $37^{\circ} \mathrm{C}$ in the humidified incubator with $5 \%$ $\mathrm{CO}_{2}$. Cells were strictly used between passage numbers 5-20 for experiments. At about $80 \%$ confluence, the cells were treated with the fresh media containing dimethyl sulfoxide (DMSO) or resveratrol. After the indicated time of treatment, the cells were harvested for further experiments. For lentiviral transduction, the virus was added with $4 \mu \mathrm{g} / \mathrm{mL}$ polybrene to cells for 4 days. Subsequently, puromycin $(4 \mu \mathrm{g} / \mathrm{mL})$ was added the day after infection for 3 days. Eventually, the drugresistant cells were gathered, expanded, and identified.

\section{Cell proliferation analysis}

To assess the cell proliferation assay, cells $\left(1 \times 10^{3}\right.$ wells $)$ were seeded in flat-bottomed 96-well plates for 5 days, and MTT assay was performed at the time point from day 1 to day 5 according to the manufacturer's protocol. All experiments were performed independently in triplicate.

\section{Annexin V-FITC/PI assay}

According to the manufacturer's instructions (Annexin VFITC/PI kit; Beyotime Institute of Biotechnology, Jiangsu, China) cells $\left(5 \times 10^{4}\right)$ were plated on coverslips. Then, $195 \mu \mathrm{L}$ 
Annexin V-FITC/PI binding buffer was added to each group, followed by $5 \mathrm{~mL}$ Annexin V-FITC/PI. After gently vortexing, all the cells were incubated in $190 \mu \mathrm{L}$ binding buffer and $10 \mu \mathrm{L}$ PI for 30 minutes without light. Twenty thousand cells for each sample were then analyzed on a FACSCalibur ${ }^{\mathrm{TM}}$ benchtop cytometer (BD Biosciences, San Jose, CA, USA) at the UW Carbone Cancer Center Flow Cytometry Facility and analyzed by FlowJo software (Tree Star, Inc., Ashland, OR, USA).

\section{5-Bromo-2'-deoxyuridine (BrdU) staining assay}

Cells were incubated with $10 \mu \mathrm{g} / \mathrm{mL}$ thymidine analog BrdU (Sigma-Aldrich Co.) in 24-well plate for 30 minutes, and then washed twice with PBS, fixed in 4\% paraformaldehyde (PFA) for 15 minutes. Afterwards, cells were permeabilized with $0.3 \%$ Triton X-100 for 5 minutes, pretreated with $1 \mathrm{~mol} / \mathrm{L} \mathrm{HCl}$ for 10 minutes, blocked with $10 \%$ goat serum for 1 hour, and then a monoclonal rat primary antibody against BrdU (1:300, ab6326, Abcam, Cambridge, UK) was incubated for 1 hour, followed by Alexa Fluor ${ }^{\circledR} 488$ goat anti-rat IgG secondary antibody, (H+L; Thermo Fisher Scientific). DAPI (300 nM) was used for nucleus staining, and the percentage of BrdU was calculated. In the end, more than eight microscopic fields were taken (Nikon 80i; Nikon Corporation, Tokyo, Japan).

\section{Plasmids and transfection}

The following human gene target-directed small interfering RNA expression vector that expresses p53-specific short hairpin RNA (shRNA) plasmids (in pLKO.1) from the RNAi Consortium were used: targeting 5'-GACTCC AGTGGTAATCTAC-3', SIRT1 shRNA, targeting 5'-AACCTTCTGTTCGGTGATGAAA-3', and green fluorescent protein (GFP)-specific shRNA (shGFP). They were purchased from GenePharma Co., Ltd (Suzhou, China). Human full-length PKM2 cDNA corresponding to amino acids (1-531) was subsequently cloned into pCDH-CMV overexpression vector to generate the recombinant plasmid using the forward primer (5'-CCGGATATCATGTCGAAGCCCCATA GTGAAG-3') in combination with the reverse primer (5'-ATA AGAATGCGGCCGCTCACGGCACAGGAACAACACGC-3'). All vectors were transfected into 293FT cells by using the Lipofectamine 2000 reagent (Thermo Fisher Scientific), subsequently, the lentivirus were infected into human melanoma cells according to the manufacturer's protocol.

\section{Antibodies and reagents}

The following antibodies and reagents were used for this study: rabbit anti-p53 (1:500, ab1431; Abcam), rabbit anti-p53 (acetyl K382) (1:1,000, ab75754; Abcam), rabbit anti-p21 (1:500, ab109520; Abcam), rabbit anti-gamma H2AX (phospho S139) (1:1,000, ab2893; Abcam), rabbit anti-ERK1/2 (1:500, No 5013; Cell Signaling Technology Inc., Danvers, MA, USA), rabbit anti-phospho-ERK1/2 (Thr202/Tyr204) (1:2,000, No 4376; Cell Signaling Technology Inc.), rabbit anti-PKM2 (1:1,000, ab150377; Abcam), rabbit anti- $\beta$-actin (1:2,000, ab16039; Abcam), mouse anti-Tubulin (1:2,000, AT819; Beyotime Institute of Biotechnology), rabbit anti-active caspase $3(1: 1,000$, ab49822; Abcam), mouse anti-cleaved PARP1 (1:1,000, ab198490; Abcam), mouse anti-cytochrome $\mathrm{C}(1: 2,000, \mathrm{ab} 13575$; Abcam), rabbit anti-VDAC (1:1,000, ab15895; Abcam), mouse anti-Bcl-2 (1:1,000, ab201566; Abcam), rabbit anti-Bax (1:500, No 2774; Cell Signaling Technology Inc.), and mouse-anti-HA (1:500, H3663; Sigma-Aldrich Co.). Resveratrol (Cat. No: R5010) was purchased from Sigma-Aldrich Co. TEPP-46 (HY18657-4) was purchased from MCE (MedChemExpress Inc., Monmouth Junction, NJ, USA).

\section{Real-time quantitative PCR (RT-qPCR)}

Total RNA was prepared by reverse transcription using Trizol reagent (Thermo Fisher Scientific) and Takara high capacity DNA synthesis kit according to the manufacturer's protocol. RT-qPCR was performed by using Power SYBR Green PCR Master Mix (TaKaRa, Shiga Prefecture, Japan) and specific primers on the CFX Connect Real-Time system (Bio-Rad Laboratories, Inc., Hercules, CA, USA). Relative mRNA levels were calculated by the $2^{-\Delta \Delta \mathrm{CT}}$ method and normalized by $\beta$-actin. The sequences of the primers used are as follows:

p53 forward: TCCTCCCCTCAATAAGCTATTCTG p53 reverse: TGGCGCTGACCCACAAC p21 forward: CGTTCACAGGTGTTTCTGCG p21 reverse: CATTAGCGCATCACAGTCGC $\beta$-Actin forward: TGGCATCCACGAGACCACCTTCA $\beta$-Actin reverse: GACTGCTGTCACCTTCACCGTTCC PKM2 forward: GCTGCCATCTACCACTTGC PKM2 reverse: CCAGACTTGGTGAGGACGATT Bax forward: TCCACCAAGAAGCTGAGCGAG Bax reverse: GTCCAGCCCATGATGGTTCT Bcl-2 forward: ATGTGTGTGGAGAGCTCAAC Bcl-2 reverse: AGCAGCCAGGAGAAATCA.

\section{Western blot analysis}

Cells were harvested and suspended in RIPA Lysis Buffer. Protein concentrations were detected with bicinchoninic acid (BCA) protein assay kit (Beyotime Institute of Biotechnology). The lysates from cells as well as the fresh tissues were 
separated by SDS-PAGE, followed by transfer onto poly vinylidene fluoride (PVDF) membranes (EMD Millipore, Billerica, MA, USA). Followed by blocking with 5\% BSA, the PVDF membranes were incubated gently with a primary antibody against the indicated human proteins at $4{ }^{\circ} \mathrm{C}$ overnight, followed by appropriate horseradish peroxidase (HRP)-conjugated secondary antibodies. HRP-labeled goat anti-mouse IgG (H+L) (A0216, 1:2,000) and goat anti-rabbit IgG $(\mathrm{H}+\mathrm{L})(\mathrm{A} 0208,1: 2,000)$ were used as secondary antibodies which were purchased from Beyotime Institute of Biotechnology. Proteins were visualized by electro-chemiluminescence (ECL) Western blot analysis system. The signal was captured by the ECL reagent (Beyotime Institute of Biotechnology) and quantitatively analyzed by densitometry using ImageJ software (developed by Wayne Rasband; National Institutes of Health, Bethesda, MD, USA).

\section{Subcellular fractionation analyses}

MV3 cells were harvested and washed thrice with cold PBS. Cytosolic or mitochondrial fractions were prepared using the ExKine Cytoplasmic Protein Extraction Kit (No KTP3003; Abbkine, Hubei, China) or the Mammalian Mitochondria Isolation Kit for Tissue and Cultured Cells (No K288; BioVision, Inc., Milpitas, CA, USA).

\section{Immunofluorescence analysis}

For immunofluorescent staining, the cells were grown on coverslips. After being fixed with 4\% PFA and permeabilized in Triton X-100, the cells were blocked with 5\% BSA and incubated with a primary antibody at $4^{\circ} \mathrm{C}$ overnight and then with the appropriate secondary antibody. The rabbit anti-gamma H2AX (phospho S139) was followed by Alexa Fluor ${ }^{\circledR} 488$ goat anti-rabbit IgG secondary antibody. The rabbit anti-PKM2 and rabbit anti-voltage-dependent anion-selective channel protein (VDAC) were followed by Alexa Fluor ${ }^{\circledR} 594$ goat anti-rabbit IgG secondary antibody. The mouse anti-cytochrome $\mathrm{c}$ and mouse anti-Bcl-2 were followed by Alexa Fluor ${ }^{\circledR} 488$ donkey anti-mouse IgG secondary antibody. In all of the above experiments, 300 nM DAPI was used for nuclear staining.

\section{Co-immunoprecipitation (co-IP) assay}

For co-IP studies, cells were lysed with IP lysis buffer. A total of $2 \mu \mathrm{g}$ PKM2 or Bcl-2 antibody as well as IgG (rabbit) was then incubated with cell lysate to pull down the PKM2 or Bcl-2 protein. After washing with PBS five times, cells were denatured at $100^{\circ} \mathrm{C}$ for 30 minutes and then separated by $10 \%$ SDS-PAGE gel. Bcl-2 or PKM2 antibody was used to check the interaction between PKM2 or Bcl-2.

\section{Ubiquitination assay}

For the ubiquitination assay, the MV3 cells were treated or transfected with indicated drugs or plasmids. At 48 hours after treatment or transfection, cells were treated with $50 \mu \mathrm{g} / \mathrm{mL}$ of proteasome inhibitor MG132 (Selleck Chemicals, Houston, TX, USA) for 6 hours. Cells were then lysed in Cell Lysis Buffer (Sigma-Aldrich Co.) for Western blot and IP following the same protocol used in co-IP. Ubiquitin antibody was used to detect the ubiquitination of Bcl-2.

\section{Glutathione S-transferase (GST) pull-down assay}

GST and GST-PKM2 proteins were transformed and expressed in Escherichia coli strain BL-21 and purified by glutathione Sepharose 4B resin (No 17075601; GE Healthcare Life Sciences China, Inc., Beijing, China). Briefly, bacterial cells $(250 \mathrm{~mL})$ were cultured in Luria broth for each construct. Protein expression was induced with $0.5 \mathrm{mM}$ (final concentration) isopropyl- $\beta$-D-thiogalactopyranoside. The proteins immobilized on the glutathione-agarose beads were quantified by Coomassie blue staining, using BSA as a protein standard.

\section{Statistical analysis}

All observations were confirmed by at least three independent experiments. Quantitative data are expressed as the mean \pm SD. A two-tailed Student's $t$-test was performed for paired samples using GraphPad Prism version 6.0 (GraphPad Software, Inc., La Jolla, CA, USA). $P<0.05$ was considered statistically significant.

\section{Result}

\section{Resveratrol inhibits melanoma cell growth and proliferation}

In this study, melanoma cell lines MV3 and A375 were treated with different concentrations of resveratrol (50, 100 , and $200 \mu \mathrm{M}$; DMSO was used as control) for 48 hours. Both MV3 and A375 cells exposed to resveratrol showed significant poor cell survival and decreased cell number in a dose-dependent manner by morphological observing (Figure 1A). Next, MTT assay confirmed that the effect of resveratrol treatment on MV3 melanoma cell viability was significant (Figure 1B). BrdU staining assay also showed that resveratrol treatment for 48 hours resulted in a significant decrease in the percentage of BrdU-positive cells compared to DMSO-treated cells (Figure 1C). These results demonstrated that resveratrol dramatically inhibited cell growth and proliferation in human melanoma cells. 


\section{Resveratrol induces apoptosis in melanoma cells}

To assess the direct anti-growth effect of resveratrol in melanoma cells, we examined DNA damage response by assessing the accumulation of $\gamma \mathrm{H} 2 \mathrm{AX}$, a marker for DNA breaks. Immunofluorescence analysis evidenced that the levels of $\gamma \mathrm{H} 2 \mathrm{AX}$ increased significantly in melanoma cells after the addition of resveratrol (Figure 1D). We then confirmed that the cell apoptosis rate was notably increased in the cells treated with different concentrations of resveratrol using flow cytometry analysis of Annexin V-FITC and PI staining (Figure 1E). Quantitative analyses of Figure 1E is

A

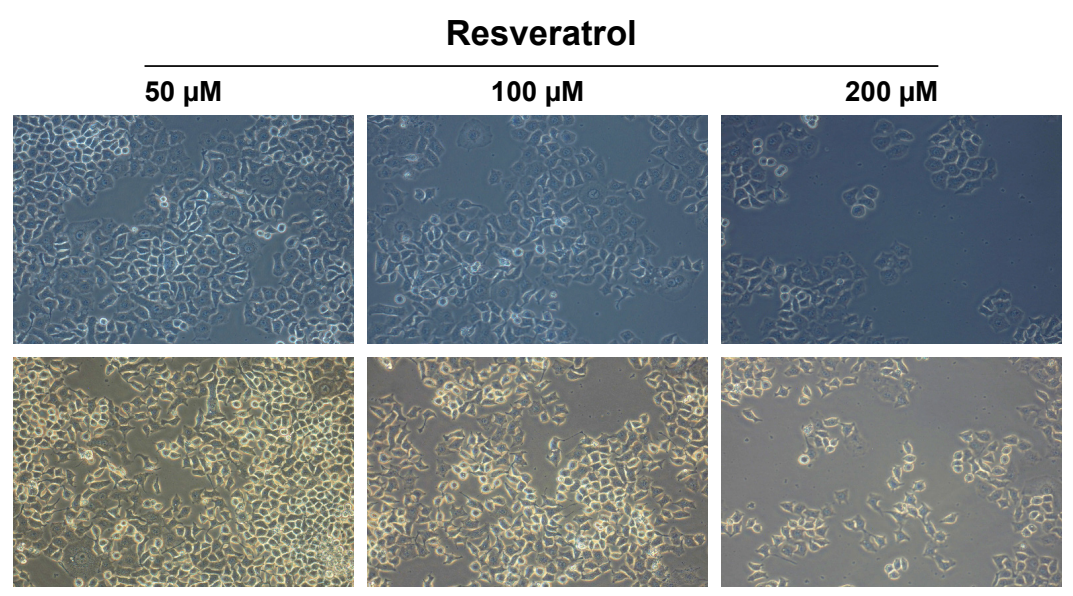

B
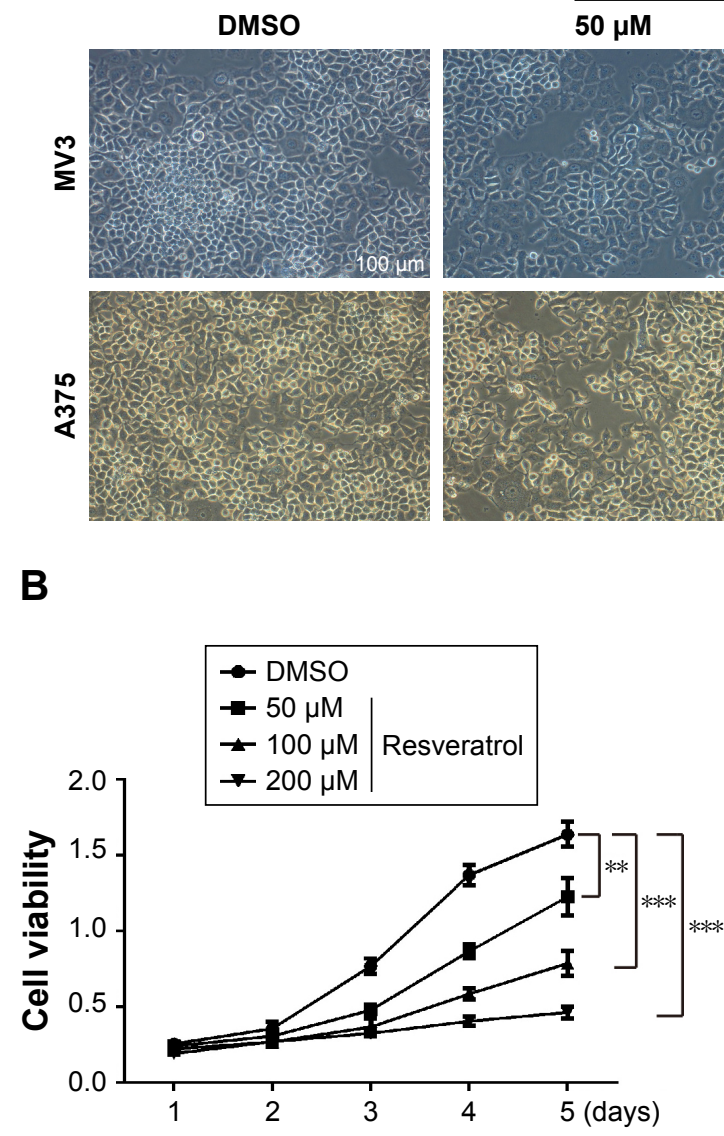

D

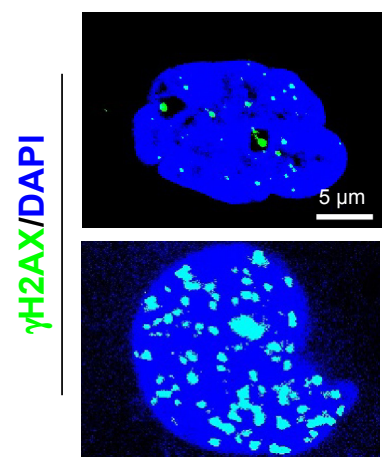

$100 \mu \mathrm{M}$
C
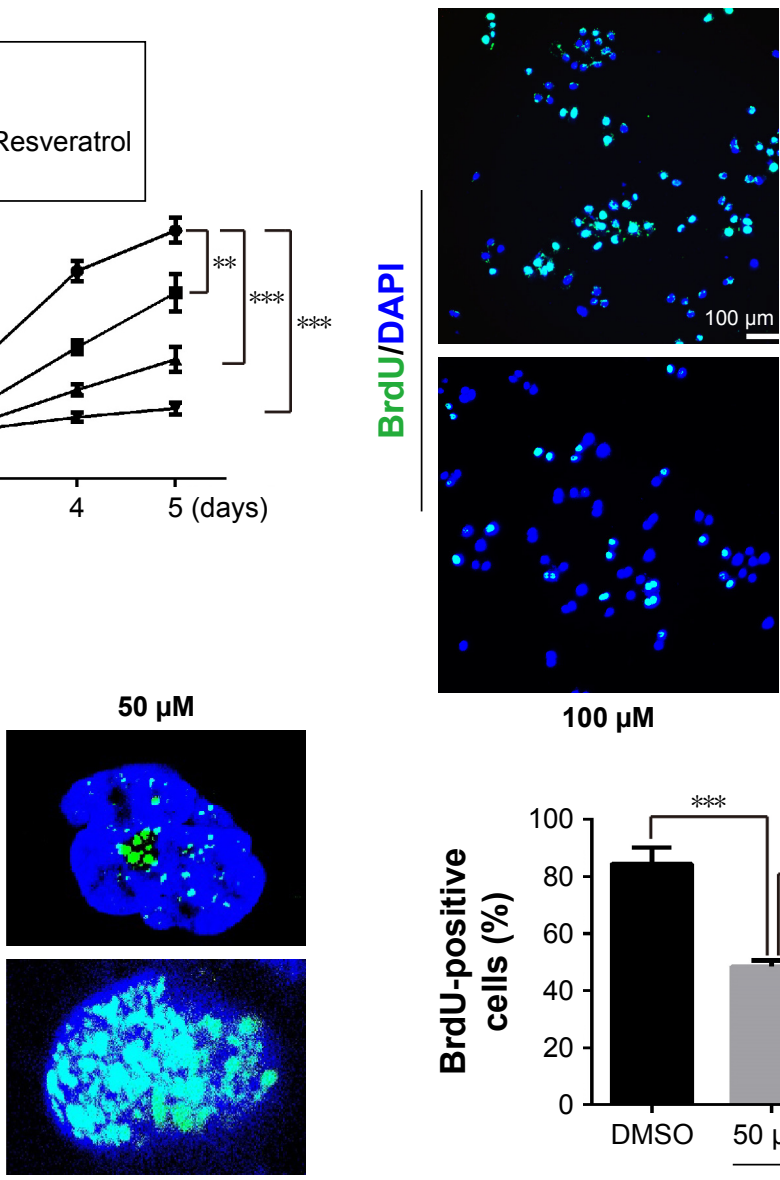

$200 \mu \mathrm{M}$
$100 \mu \mathrm{M}$

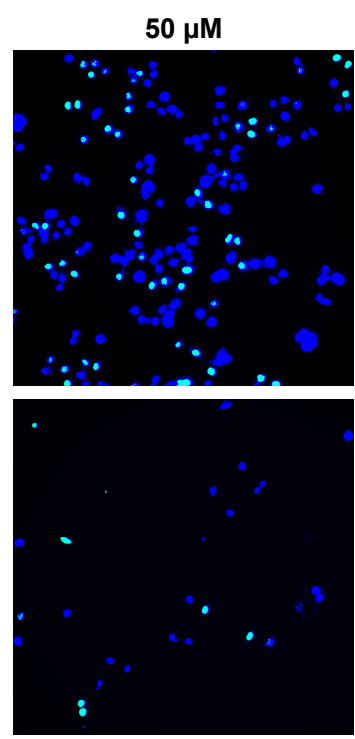

$200 \mu \mathrm{M}$

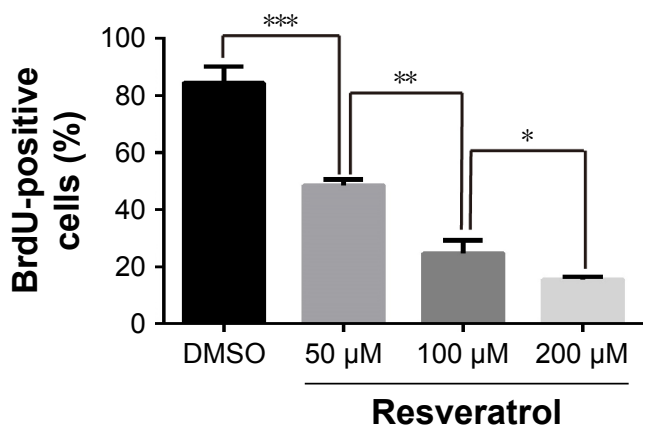

Figure I (Continued) 

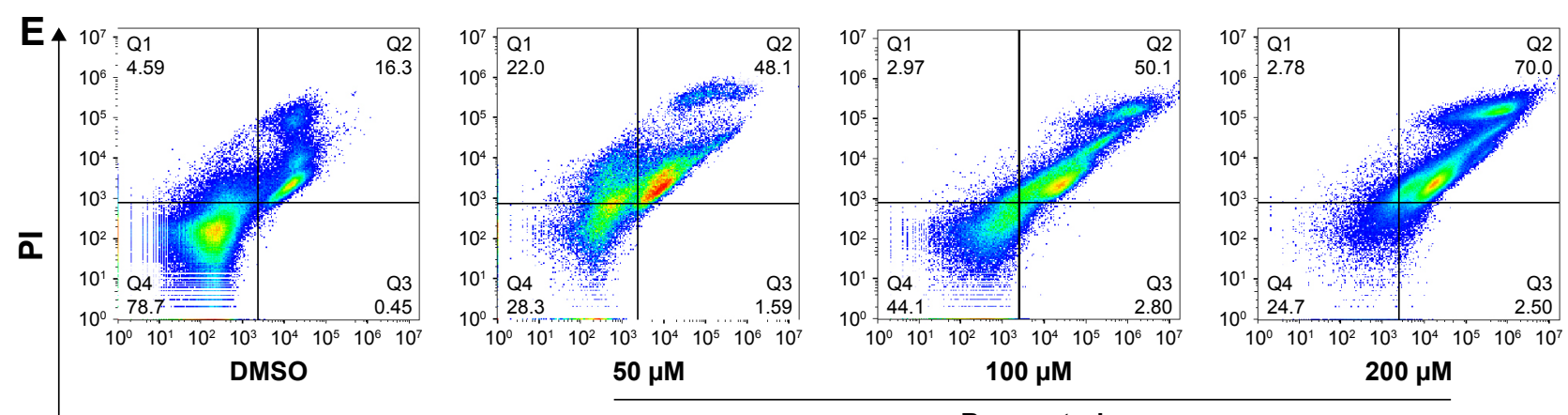

\section{Resveratrol}

\section{Annexin V-FITC}
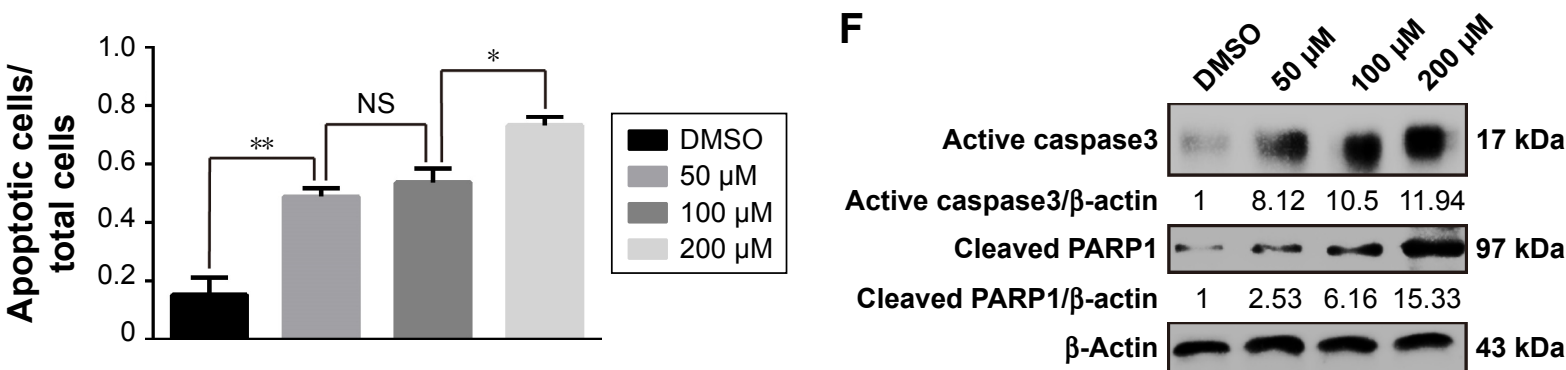

Figure I Resveratrol inhibited the proliferation and induced apoptosis in human melanoma cells.

Notes: (A) Cell morphology of MV3 and A375 melanoma cells after treating with the indicated concentration of resveratrol for 48 hours; scale bar as shown. Treatment with DMSO was used as control. (B) MTT assay was performed to examine the effect of indicated concentration of resveratrol on cell viability. All data were shown as the mean $\pm S D, * P<0.05, * * P<0.01$, $* * * P<0.00$ I, and NS $P \geq 0.05$. (C) Image and quantification of MV3 cells positive for BrdU staining after treating as in (B); scale bar as shown. (D) Immunofluorescence analysis to identify $\gamma \mathrm{H} 2 \mathrm{AX}$-positive nuclei in MV3 cells treated as in (B). (E) Apoptosis of MV3 cells treated as in (B). Cells were stained with PI and Annexin V-FITC, and the positively stained cells were counted using flow cytometry. Quantification of apoptotic cells is presented on the lower left. (F) Western blot analysis was performed for evaluating the activation of cleaved Caspase 3 and expression of cleaved PARPI in MV3 cells treated as in (B). $\beta$-Actin acts as loading control. Densitometry of Western blot in the bottom of each panel is shown as the ratio to $\beta$-actin.

Abbreviation: BrdU, 5-bromo-2'-deoxyuridine; DMSO, dimethyl sulfoxide; FITC, fluorescein isothiocyanate; NS, not significant; PI, propidine iodide.

shown on the lower left and illustrated that the cell apoptosis rate was dramatically increased by up to $73.3 \%$ in $200 \mu \mathrm{M}$ resveratrol-treated cells (Figure 1E). To further confirm these results, we analyzed the expression levels of several apoptosis-associated proteins using Western blot. Active Caspase 3 and cleaved PARP1 were increased in resveratroltreated cells in a dose-dependent manner (Figure 1F). These results demonstrated that resveratrol treatment could induce melanoma cell apoptosis in vitro.

\section{Block of p53 fails to recover the proliferation ability inhibited by resveratrol}

During the experiments, we found that an important apoptotic protein $\mathrm{p} 53$ and its downstream effector $\mathrm{p} 21$ expressions were significantly increased in both mRNA and protein levels in MV3 cells (Figure 2A-C). To gain insight into the mechanisms underlying the role in p53 accumulation in resveratrol-treated cells, we examined levels of p53 hyperacetylation. However, no p53 hyperacetylation was observed indicating that activation of $\mathrm{p} 53$ pathway might not be resveratrol-induced apoptosis dependent. To confirm whether p53 upregulation had the necessary connection with resveratrol-induced apoptosis, resveratrol-treated MV3 cells were then stably infected with a lentivirus system carrying shRNA against $p 53$ gene. qRT-PCR and Western blot suggested that $\mathrm{p} 53$ and $\mathrm{p} 21$ were simultaneously downregulated after infection in both mRNA and protein levels compared to resveratrol treatment alone (Figure 2D-F). Nevertheless, the proteins levels of active Caspase 3 and cleaved PARP1 were not decreased significantly after p53 knockdown, indicating that apoptosis might persist (Figure 2F). Immunofluorescence of $\gamma \mathrm{H} 2 \mathrm{AX}$ and BrdU assay further showed that downregulation of $\mathrm{p} 53$ failed to restore DNA damage and cell apoptosis induced by resveratrol treatment (Figure $2 \mathrm{G}$ and $\mathrm{H}$ ). These results demonstrated that resveratrol-induced cell proliferation inhibition and apoptosis were independent of $\mathrm{p} 53$ regulation, revealing that resveratrol caused another apoptotic effector distinct from p53 pathway. 

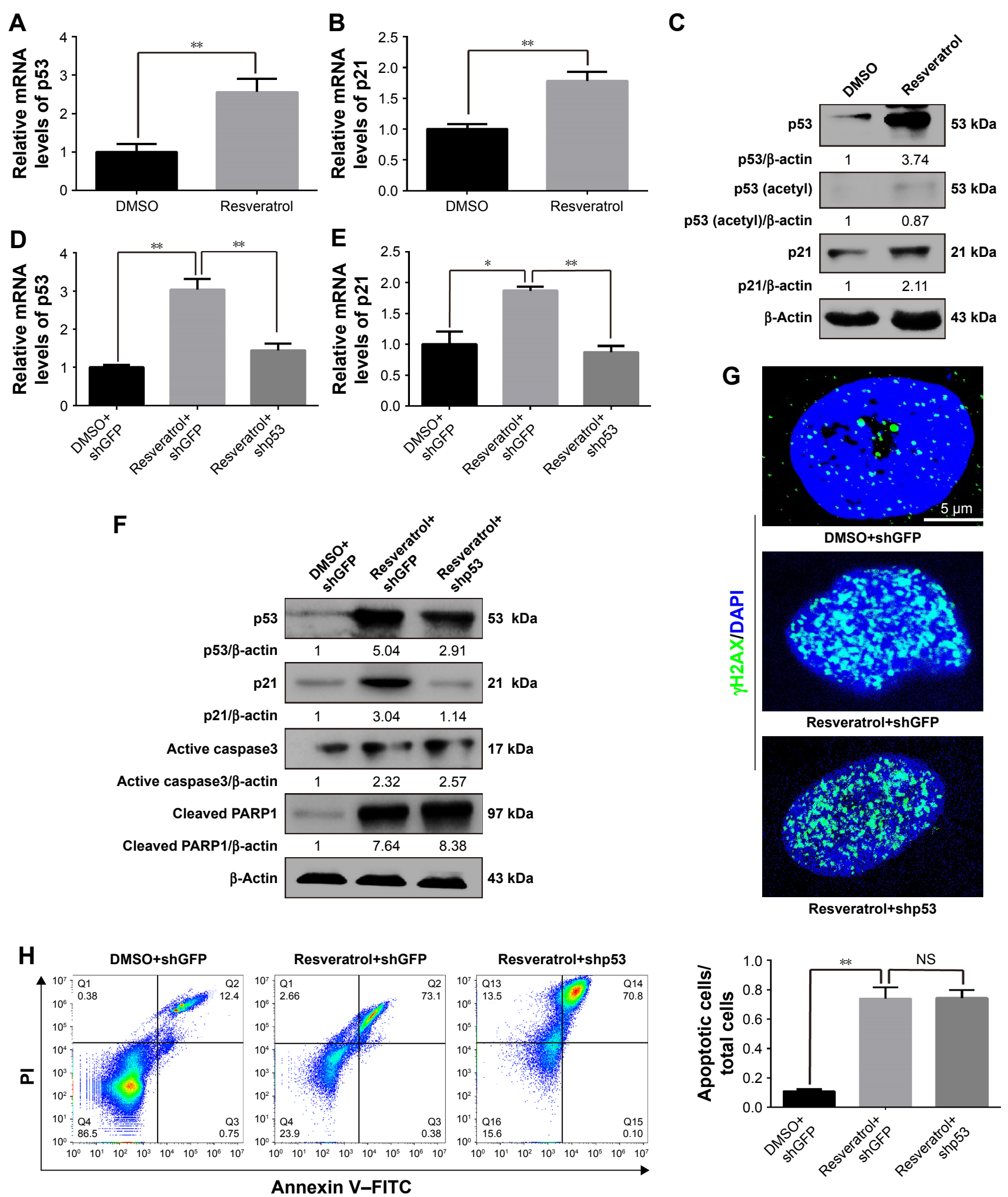

Figure 2 Resveratrol-induced apoptosis was independent of the p53-mediated pathway in human melanoma cells.

Notes: (A and B) MV3 cells were treated with $200 \mu \mathrm{M}$ resveratrol or DMSO (as control) for 48 hours. RNA was isolated for performing qPCR using $\beta$-actin as reference gene to determine $p 53$ and $p 21$ mRNA levels. All data were shown as the mean \pm SD, $* P<0.05, * * P<0.01$, and NS $P \geq 0.05$. (C) Western blot analysis of the protein levels of $\mathrm{p} 53$ and acetylated $\mathrm{p} 53$ and $\mathrm{p} 2 \mathrm{I}$ in extracts prepared from MV3 cells treated with $200 \mu \mathrm{M}$ resveratrol or DMSO. Expression of $\beta$-actin was used as a loading control. (D and E) mRNA levels of $p 53$ and $p 2 I$ in MV3 cells treated with DMSO or resveratrol-transduced with indicated shRNA for p53/GFP by RT-qPCR were analyzed. (F) The expression levels of p53, p2I, active caspase3, and cleaved PARPI were determined using Western blot analysis after cells were treated as in (D). (G) Immunofluorescence analysis to identify $\gamma \mathrm{H} 2 \mathrm{AX}$-positive nuclei in MV3 cells treated as in (D). (H) Apoptosis was analyzed in MV3 cells treated as in (D). Quantification of apoptotic cells is presented on the lower right.

Abbreviations: DAPI, diamidine phenylindole; DMSO, dimethyl sulfoxide; GFP, green fluorescent protein; NS, not significant; RT-qPCR, real-time quantitative PCR; shGFP, GFP-specific shRNA; shRNA, short hairpin RNA. 


\section{Resveratrol induces $\mathrm{Bcl}-2$ degradation and mitochondria-dependent apoptosis}

Based on previous results, we hypothesized that resveratrol might induce apoptosis by downregulating antiapoptotic Bcl-2 expression in melanoma cells. However, mRNA levels of Bcl-2 revealed no significant difference between shGFP and shp53 MV3 cells with or without $200 \mu \mathrm{M}$ resveratrol treatment, respectively, for 48 hours, suggesting that resveratrol might posttranscriptionally regulate $\mathrm{Bcl}-2$, whereas only mRNA levels of Bax (Bcl-2 family member) were markedly increased in shp53 MV3 cells treated with resveratrol (Figure $3 \mathrm{~A}$ and $\mathrm{B}$ ). By contrast, the protein level of Bcl-2 was obviously downregulated in shp53 MV3 cells with or without $200 \mu \mathrm{M}$ resveratrol treatment as expected, meanwhile, the protein level of Bax was conversely markedly upregulated (Figure 3C). To further investigate the impact of resveratrol on Bcl-2 stability, we examined the ubiquitination of Bcl-2 and found that resveratrol reduced the ubiquitination levels of Bcl-2 in resveratrol-treated cells (Figure 3D).

In the intrinsic pathway, Bcl-2-mediated pathway could induce cytochrome $\mathrm{c}$ release from the mitochondria to the cytosol to result in mitochondria-dependent apoptosis. ${ }^{18}$ More cytochrome $\mathrm{c}$ were detected in cytosolic fraction in resveratrol-treated MV3 cells than that in the control cells (Figure 3E), which was supported by immunofluorescent analysis of subcellular localization between cytochrome $\mathrm{c}$ and VDAC (expressed and located in the outer mitochondrial membrane) showing cytochrome c release during resveratrol-induced apoptosis stage (Figure 3F). These results demonstrated that resveratrol induces mitochondriadependent apoptosis by regulating Bcl-2 stability through upregulation of the levels of Bcl-2 ubiquitination.

\section{Resveratrol suppresses PKM2, which interacts with and stabilizes $\mathrm{Bcl}-2$}

Previous studies have shown that PKM2 regulates the stability of Bcl-2 through inhibiting ubiquitination. ${ }^{18}$ In order to reveal whether PKM2 was involved in apoptotic effects induced by resveratrol, we performed Western blot analysis and found that PKM2 expression was negatively regulated by resveratrol in both mRNA and protein levels, which was also the case with phosphorylated Erk $1 / 2$ located in the upstream of PKM2 (Figure 4A and B). To investigate the physical interaction between PKM2 and Bcl-2 in MV3 cells, we performed co-IP assay and found that PKM2 associated with Bcl-2 in vivo reciprocally (Figure 4C). These observations were further supported by an immunofluorescence staining, showing that PKM2 colocalized with Bcl-2 after resveratrol treatment (Figure 4D).

TEPP-46 treatment blocked PKM2 activity by triggering PKM2 change from dimerization into tetramerization and decreasing PKM2 methylation. ${ }^{19}$ To confirm whether PKM2 tetramer formation or methylation regulated the interaction with Bcl-2 under resveratrol treatment, we performed GST pull-down using recombinant GST-tagged PKM2. The PKM2-Bcl-2 interaction was observed under both resveratrol and TEPP-46 treatments, indicating neither tetramer formation nor methylation in PKM2 affected direct interaction with Bcl-2 (Figure 4E).

To further confirm that PKM2 had a biological impact on Bcl-2, we performed MV3 cells infected with lentiviruses encoding PKM2. Both qRT-PCR and Western blot assay showed that the expression of PKM2 was rescued in the resveratrol-treated cells (Figure 4F and G). Furthermore, we found that Bcl-2 protein level was restored in PKM2overexpressed MV3 cells, indicating reduced Bcl-2 degradation under resveratrol treatment (Figure 4G). These results demonstrated that ubiquitin-dependent degradation of Bcl-2 caused by resveratrol could be rescued by restoring the expression of PKM2.

\section{Overexpression of PKM2 rescues resveratrol-induced cell proliferation inhibition and apoptosis in human melanoma cells}

As shown in Figure 4H and I, BrdU assay and apoptosis analysis showed that overexpressing PKM2 promoted cell proliferation, and dramatically decreased apoptosis induced by resveratrol. These results demonstrated that resveratrol might inhibit proliferation and induce apoptosis by targeting PKM2 in melanoma cells.

\section{Discussion}

Resveratrol is one of the most promising antitumor drugs to inhibit cancer initiation, progression, and metastasis. ${ }^{14,17} \mathrm{Cer}-$ tainly, there are many studies that indicated that resveratrol can induce apoptosis in melanoma cells by inducing the expression of proapoptotic proteins. ${ }^{10}$ Various apoptosis signaling pathways are involved in resveratrol-mediated mechanisms but the specific target impacted by resveratrolinduced apoptosis remains unclear.

Here, we demonstrated p53-related apoptosis was obvious in resveratrol-treated melanoma cells but not unique and dependent for resveratrol-induced apoptosis, which was in 

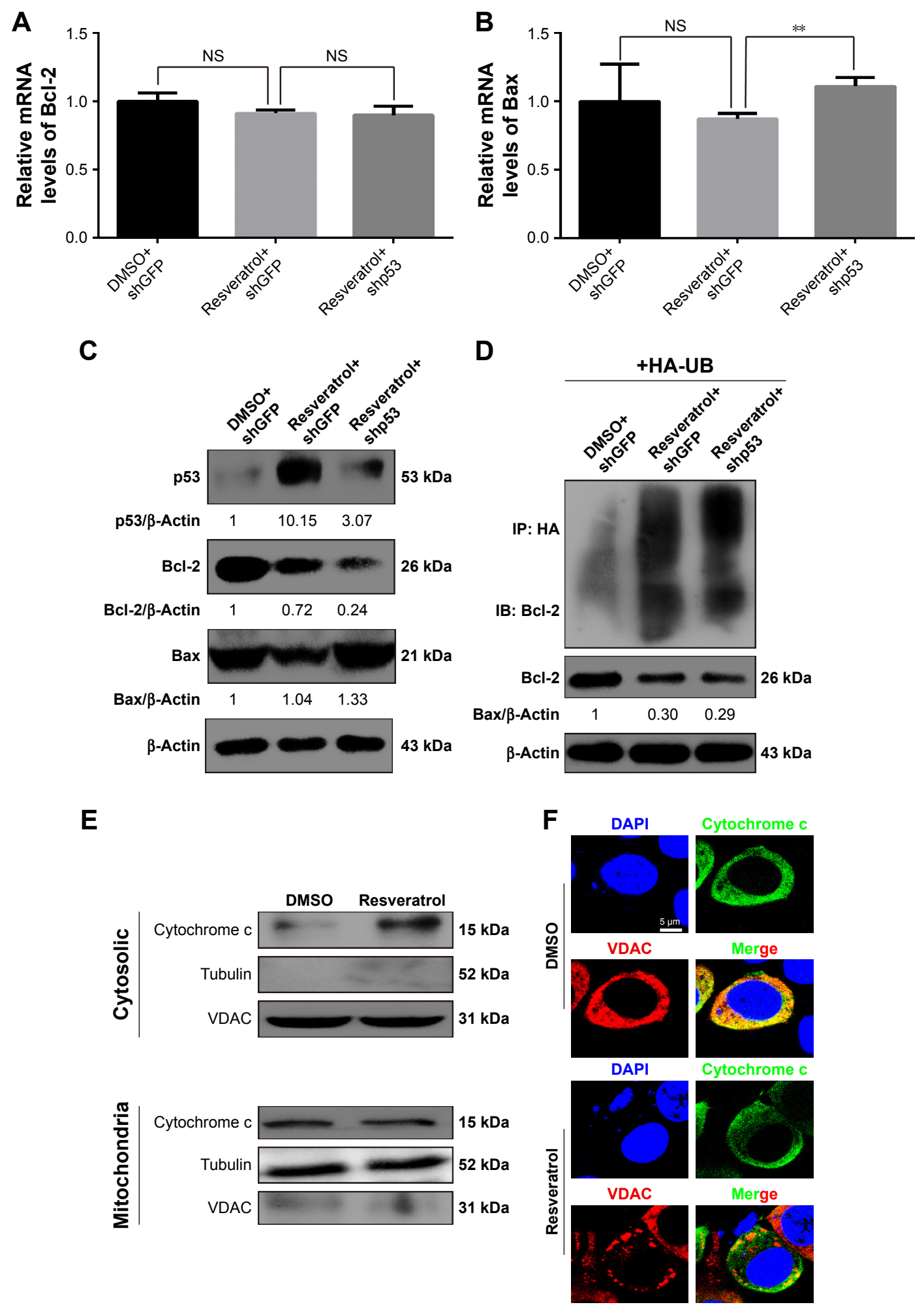

Figure 3 Effect of resveratrol involved in degradation of $\mathrm{Bcl}-2$ and release of cytochrome $\mathrm{c}$ in human melanoma cells.

Notes: (A and B) shGFP- or shp53-transduced MV3 cells were treated with or without resveratrol (200 $\mu \mathrm{M}, 48$ hours). A qRT-PCR was performed to examine mRNA levels of $\mathrm{BCl}-2$ and Bax. All data were shown as the mean $\pm S D$, $* * P<0.01$, and $\mathrm{NS} P \geq 0.05$. (C) The expressions of $\mathrm{p} 53, \mathrm{BCl}-2$, and Bax were checked in shp53-transduced MV3 cells treated with or without $200 \mu \mathrm{M}$ resveratrol for 48 hours. DMSO and shGFP vector were used as control. $\beta$-Actin was used as internal reference. (D) Resveratrol-treated and/or shp53transduced MV3 cells were treated with MGI32 for 6 hours before harvesting. The ubiquitinated Bcl-2 proteins were pulled down with anti-HA antibody and immunoblotted with anti-Bcl-2 antibody using streptavidin agarose beads. (E) MV3 cells treated with or without resveratrol were harvested for cytosolic (Cyto) and mitochondrial (Mito) fractionation. Western blot assay was used to show the expression levels of cytochrome $\mathrm{c}$ in cytosolic or mitochondrial fraction. VDAC and Tublin were used as loading control. (F) Immunofluorescence analyses were performed using anti-cytochrome $\mathrm{c}$ or anti-VDAC antibodies in MV3 cells treated with or without resveratrol. Scale bar as shown. Abbreviations: DAPI, diamidine phenylindole; DMSO, dimethyl sulfoxide; IB, immunoblotting; IP, immunoprecipitation; NS, not significant; qRT-PCR, real-time quantitative PCR; shGFP, GFP-specific shRNA; VDAC, voltage dependent anion channel protein. 
agreement with our previous reports suggesting the existence of other mechanisms of resveratrol-related apoptosis regulation in human melanoma cells. ${ }^{11,12}$ Furthermore, our results revealed that resveratrol induced melanoma cell apoptosis by degrading Bcl-2 rather than upregulating p53. Next, attenuated Bcl-2 lost its inhibition of Bax and then induced the release of cytochrome $\mathrm{c}$ into the cytoplasm, which caused apoptosis. Finally, overexpression of PKM2

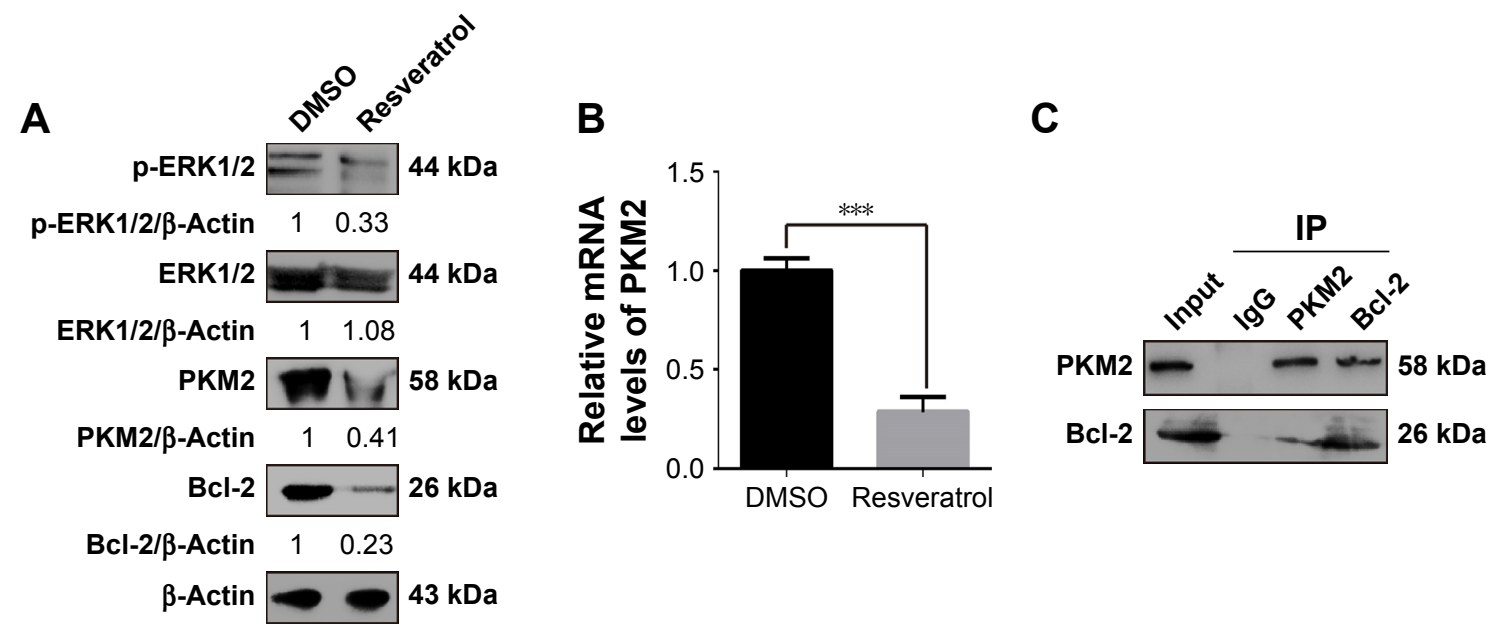

D

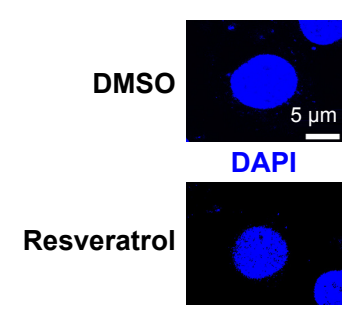

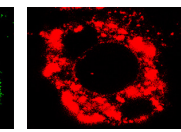

PKM2
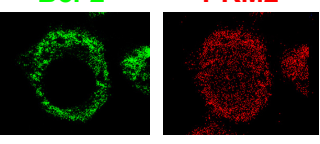

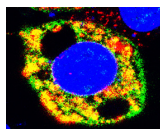

Merge

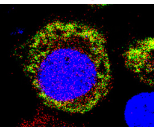

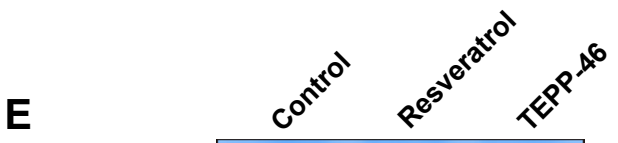

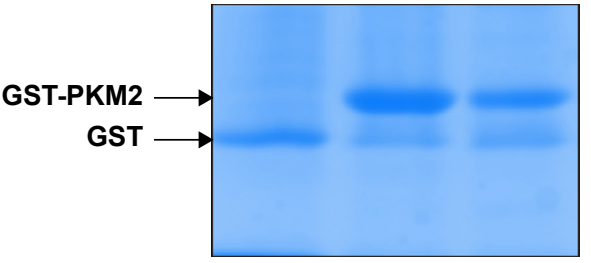

Coomassie staining

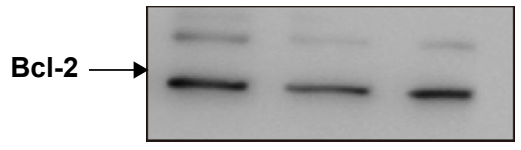

Autoradiograph

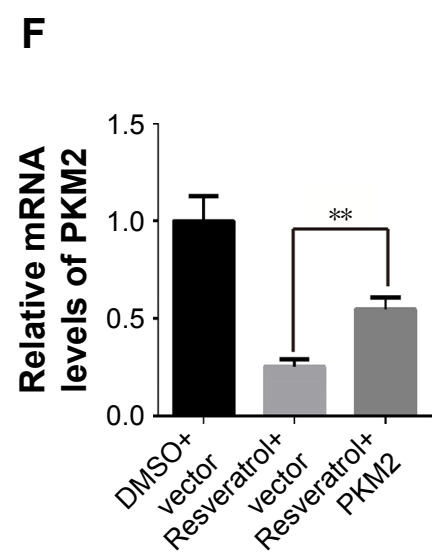

G

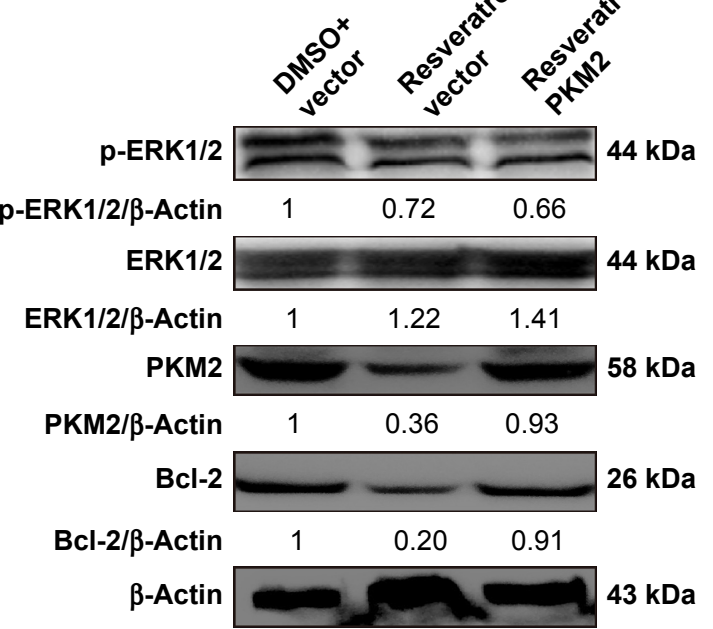

J

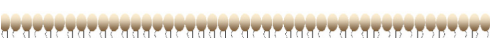

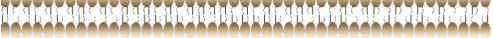

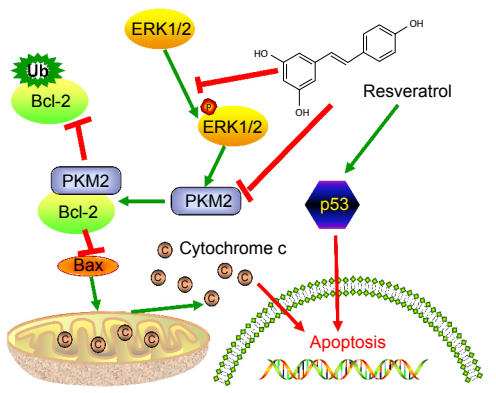

Figure 4 (Continued) 
H

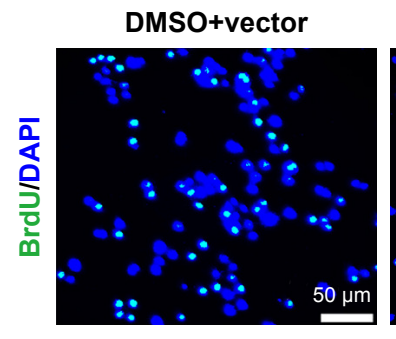

Resveratrol+vector
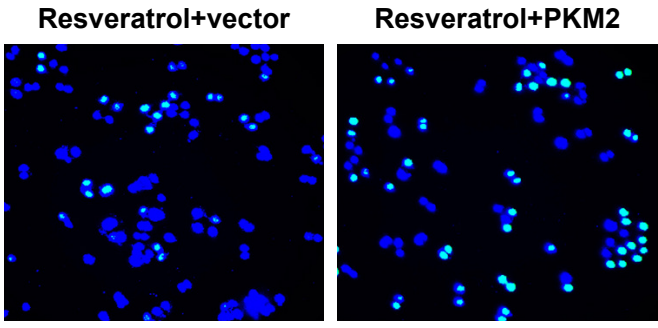

I
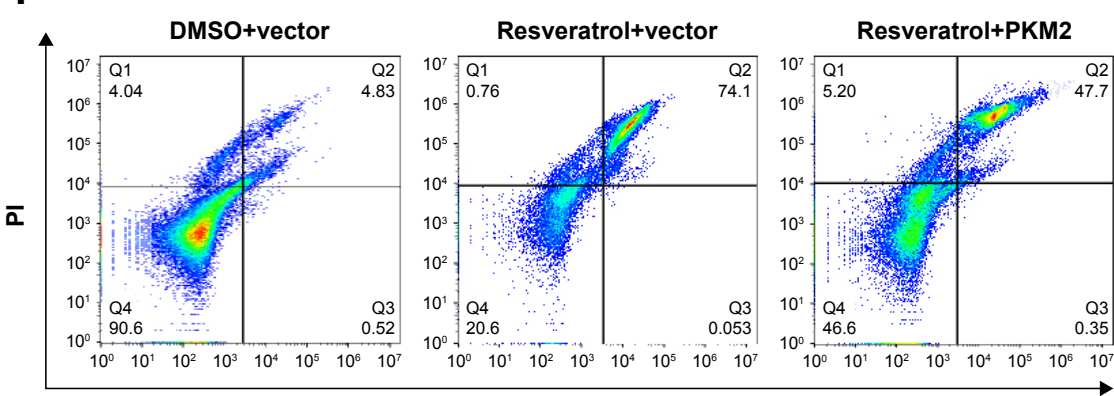

Annexin V-FITC
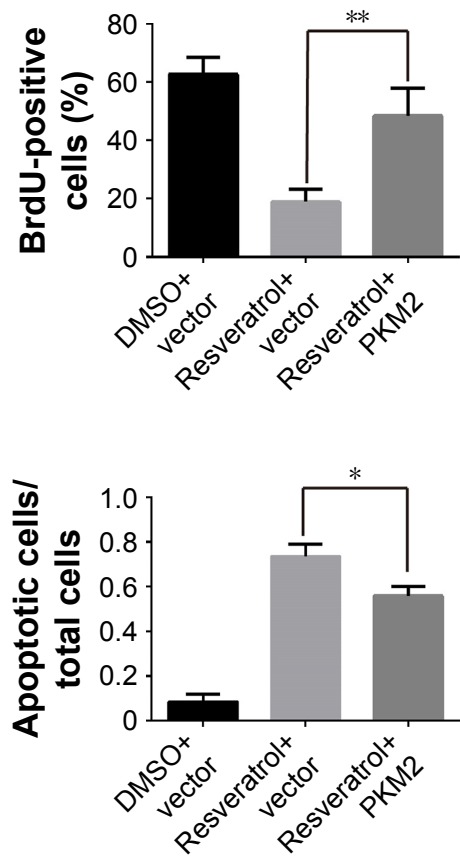

Figure 4 PKM2 interacted with $\mathrm{Bcl}-2$ and resisted resveratrol-induced apoptosis in human melanoma cells.

Notes: (A) Western blots showing the expression of P-ERKI/2, ERKI/2, PKM2, and Bcl-2 in MV3 cells after treating with $200 \mu M$ resveratrol for 48 hours. (B) RNA was extracted from MV3 cells after treating with $200 \mu \mathrm{M}$ resveratrol for 48 hours and qPCR was performed using indicated primers for analysis of $P K M 2$. All data were shown as the means $\pm S D, * P<0.05$, $* * P<0.01$, and NS $P \geq 0.05$. (C) Reciprocal co-IP of PKM2 and Bcl-2 in MV 3 melanoma cells followed by Western blot analysis. IgG represents a control antibody used for IPs. Five hundred micrograms of lysates were used for each IP reaction and $50 \mu g$ lysates were used as input controls. (D) Immunofluorescence was performed using anti-PKM2 and anti-Bcl-2 antibodies in MV3 cells after treating with $200 \mu M$ resveratrol for 48 hours. Scale bar as shown. (E) Direct interaction of recombinant GST-PKM2 protein with Bcl-2 in GST pull-down. GST-tagged PKM2 proteins purified from MV3 cells after treating with resveratrol $(200 \mu \mathrm{M})$ or TEPP-46 (I $\mu \mathrm{M})$ for 48 hours were detected by anti-Bcl-2 antibody (bottom), and protein loading was detected by CBB staining after resolution by $8 \%$ SDS-PAGE (top). (F) mRNA levels of PKM2 were analyzed by qPCR in PKM2 overexpressing MV3 cells that were treated with resveratrol as indicated. DMSO and empty vector were used as controls. (G) The expression levels of indicated proteins were analyzed using Western blot assays in PKM2 overexpressing and control cells that were treated with resveratrol as indicated. $\beta$-Actin was used as a loading control. $(\mathbf{H})$ Image and quantification of PKM2-overexpressed MV3 cells after treating with resveratrol as well as control cells positive for BrdU staining. Scale bar as shown. (I) Apoptosis was analyzed in MV3 overexpressing with Bcl after treating with resveratrol as indicated. DMSO and empty vector were used as controls. Quantification of apoptotic cells is presented on the lower right. (J) Schema illustrating the mechanism of regulation of resveratrol on PKM2-mediated $\mathrm{Bcl}$-2 stabilization and apoptosis resistance in human melanoma cell.

Abbreviations: CBB, coomassie brilliant blue; co-IP, co-immunoprecipitation; DAPI, diamidine phenylindole; DMSO, dimethyl sulfoxide; GST, glutathione S-transferase; PKM2, M2-type pyruvate kinase.

in resveratrol-treated melanoma cells indicated that resveratrol had anti-melanoma ability through targeting Erk1/2 and PKM2, meanwhile PKM2 acted as the key in stabilizing $\mathrm{Bcl}-2$ protein level. In brief, PKM2-/Bcl-2-independent regulations would contribute to resveratrol-induced melanoma cells apoptosis in a specific manner (Figure 4J).

As known, PKM2 has contributed to regulating cancer metabolism and tumorigenesis..$^{18,20,21}$ As per our previous researches, nicotinamide phosphoribosyl transferase (NAMPT)/ E2F2/SIRT1 axis catalyzes an alternative $\mathrm{NAD}^{+}$salvage pathway leading to reduced susceptibility to NAMPT inhibitors, along with the decreased expression of PKM2 in the mRNA and protein levels. ${ }^{11,20}$ However, little is known about the direct or indirect correlation between $\mathrm{NAD}^{+}$-dependent biological events and the epigenetic mechanism of PKM2 in melanoma. It's very interesting to highlight the link and interaction between PKM2 epigenetic regulation and NAMPT-dependent signal pathway in the process of proliferation and metabolism.
Taken together, the findings gathered in this study not only contribute to better understand the therapeutic mechanism of resveratrol in melanoma cells but also provide strong support that targeting Erk/PKM2/Bcl-2 is a novel valuable clinical option as a new antimetabolic route for innovative antineoplastic chemicals to treat malignant melanoma. Our future research will involve studies based on the exploration of new targets, directly and indirectly, related to both the alternative $\mathrm{NAD}^{+}$salvage pathway in the NAMPT/E2F2/SIRT1 axis and the downstream signaling regulated by PKM2 epigenetic regulation.

\section{Acknowledgment}

This work was supported in part by National Natural Science Foundation of China (No 31701007), Training Project of Young Talents Engineering of Science and Education of Guizhou Province (No 2017-198), the Initial Research Funds for the PhD in Zunyi Medical University (F-818), Students' 
innovation and entrepreneurship training program of Guizhou Province (No 201710661042) and Students' innovation and entrepreneurship training program of Zunyi Medical University (Nos Zunyi201751021 and Zunyi201753808).

\section{Disclosure}

The authors report no conflicts of interest in this work.

\section{References}

1. Rastrelli M, Tropea S, Rossi CR, Alaibac M. Melanoma: epidemiology, risk factors, pathogenesis, diagnosis and classification. In Vivo. 2014; 28(6):1005-1011.

2. Hu H, Dong Z, Tan P, et al. Antibiotic drug tigecycline inhibits melanoma progression and metastasis in a p21CIP1/Waf1-dependent manner. Oncotarget. 2016;7(3):3171-3185.

3. Vijuk G, Coates AS. Survival of patients with visceral metastatic melanoma from an occult primary lesion: a retrospective matched cohort study. Ann Oncol. 1998;9(4):419-422.

4. Neila J, Soyer HP. Key points in dermoscopy for diagnosis of melanomas, including difficult to diagnose melanomas, on the trunk and extremities. J Dermatol. 2011;38(1):3-9.

5. Veierød MB, Weiderpass E, Thörn M, et al. A prospective study of pigmentation, sun exposure, and risk of cutaneous malignant melanoma in women. J Natl Cancer Inst. 2003;95(20):1530-1538.

6. Zhao Y, He J, Li J, et al. Demethylzeylasteral inhibits cell proliferation and induces apoptosis through suppressing MCL1 in melanoma cells. Cell Death Dis. 2017;8(10):e3133.

7. Kaufmann SH, Gores GJ. Apoptosis in cancer: cause and cure. Bioessays. 2000;22(11):1007-1017.

8. Serrone L, Hersey P. The chemoresistance of human malignant melanoma: an update. Melanoma Res. 1999;9(1):51-58.

9. Soengas MS, Lowe SW. Apoptosis and melanoma chemoresistance. Oncogene. 2003;22(20):3138-3151.

10. de Oliveira MR, Nabavi SF, Manayi A, Daglia M, Hajheydari Z, Nabavi SM. Resveratrol and the mitochondria: From triggering the intrinsic apoptotic pathway to inducing mitochondrial biogenesis, a mechanistic view. Biochim Biophys Acta. 2016;1860(4):727-745.
11. Zhao H, Tang W, Chen X, et al. The NAMPT/E2F2/SIRT1 axis promotes proliferation and inhibits p53-dependent apoptosis in human melanoma cells. Biochem Biophys Res Commun. 2017;493(1):77-84.

12. Zhao H, Yang L, Cui H. SIRT1 regulates autophagy and diploidization in parthenogenetic haploid embryonic stem cells. Biochem Biophys Res Commun. 2015;464(4):1163-1170.

13. Kala R, Shah HN, Martin SL, Tollefsbol TO. Epigenetic-based combinatorial resveratrol and pterostilbene alters DNA damage response by affecting SIRT1 and DNMT enzyme expression, including SIRT1dependent $\gamma-\mathrm{H} 2 \mathrm{AX}$ and telomerase regulation in triple-negative breast cancer. BMC Cancer. 2015;15:672.

14. Wu H, Wang Y, Wu C, Yang P, Li H, Li Z. Resveratrol induces cancer cell apoptosis through MiR-326/PKM2-Mediated ER stress and mitochondrial fission. J Agric Food Chem. 2016;64(49):9356-9367.

15. Zhang D, Wang F, Pang Y, et al. Down-regulation of CHERP inhibits neuroblastoma cell proliferation and induces apoptosis through ER stress induction. Oncotarget. 2017;8(46):80956-80970.

16. Yang W, Zheng Y, Xia Y, et al. ERK1/2-dependent phosphorylation and nuclear translocation of PKM2 promotes the Warburg effect. Nat Cell Biol. 2012;14(12):1295-1304.

17. Wu H, He L, Shi J, et al. Resveratrol inhibits VEGF-induced angiogenesis in human endothelial cells associated with suppression of aerobic glycolysis via modulation of PKM2 nuclear translocation. Clin Exp Pharmacol Physiol. 2018;45(12):1265-1273.

18. Liang J, Cao R, Wang X, et al. Mitochondrial PKM2 regulates oxidative stress-induced apoptosis by stabilizing Bcl2. Cell Res. 2017;27(3): 329-351.

19. Liu F, Ma F, Wang Y, et al. PKM2 methylation by CARM1 activates aerobic glycolysis to promote tumorigenesis. Nat Cell Biol. 2017;19(11): 1358-1370.

20. Wu H, Yang P, Hu W, et al. Overexpression of PKM2 promotes mitochondrial fusion through attenuated p53 stability. Oncotarget. 2016; 7(47):78069-78082.

21. Dong G, Mao Q, Xia W, et al. PKM2 and cancer: The function of PKM2 beyond glycolysis. Oncol Lett. 2016;11(3):1980-1986.
OncoTargets and Therapy

\section{Publish your work in this journal}

OncoTargets and Therapy is an international, peer-reviewed, open access journal focusing on the pathological basis of all cancers, potential targets for therapy and treatment protocols employed to improve the management of cancer patients. The journal also focuses on the impact of management programs and new therapeutic agents and protocols on

\section{Dovepress}

patient perspectives such as quality of life, adherence and satisfaction. The manuscript management system is completely online and includes a very quick and fair peer-review system, which is all easy to use. Visit http://www.dovepress.com/testimonials.php to read real quotes from published authors. 